\title{
An odometry free automatic perpendicular parking strategy for a light urban vehicle based on a low resolution lidar
}

\author{
Giulio Panzani, Dario Nava and Sergio M. Savaresi
}

\begin{abstract}
In this paper, an automatic perpendicular parking algorithm for a light urban vehicle with active steer assembly is presented, designed to help the user when the manoeuvre has to be performed in a narrow space between two obstacles. The parking strategy is odometry free and based only on the measurements from a limited FOV low resolution Lidar, resulting in a simple and cost effective solution. In the initial phase of the manoeuvre, the algorithm exploits the Lidar information combined with the a priori knowledge of the park structure - to localize the vehicle in the parking environment and to generate the steering action accordingly, so as to approach the parking space. Then, as the vehicle approaches the parking spot entrance, an obstacle avoidance logic is applied to end the manoeuvre without crashes. The performance of the proposed strategy has been assessed through an extensive simulation campaign and validated by experimental tests using a real instrumented vehicle.
\end{abstract}

\section{INTRODUCTION}

In the Advanced Driver Assistance Systems (ADAS) context, automatic parking represents one of the research area of major interest due to the multiple advantages introduced by this technology. Related benefits range from the enhancement of the driver comfort to safety improvements, due to the effectiveness of such systems in perform collision-free manoeuvres in environments characterized by the possible presence of obstacles.

In literature, a wide number of examples of autonomous parking systems for car-like vehicles can be found, based on the information retrieved from different sensors - from low cost setups comprising cameras and ultrasonic sensors, to high end systems based on Lidar and radar scanners - and exploiting a great variety of planning and control techniques. Two comprehensive surveys on the topic can be found in the recent works [1] and [2].

Focusing on the low cost solutions, the most common setup choice to perform automatic parking manoeuvres is the employment of ultrasonic sensors - which can be typically found on vehicles on the market - to explore the surrounding environment and detect obstacles. Due to the poor angular resolution of the measurements, usually parking algorithms based on such sensing systems exploit the following steps.

All the authors are with Dipartimento di Elettronica, Informazione e Bioingegneria, Politecnico di Milano, Italy.

Corresponding author: giulio.panzani@polimi.it
Firstly, the parking area is detected, and the relative pose between the vehicle and the parking spot reconstructed. One common approach, especially for parallel parking, is to scan and map the parking area combining distance measures and odometry, taking advantage of a low speed transit next to it, see e.g. [3]-[6]. Alternatively, the detection can be made directly combining distance measures with additional information retrieved from a camera sensor [7]-[9], without the need for an initial scanning. At this point, to perform the parking manoeuvre, two approaches are possible. The first one exploits a path planning phase, where the best path for the vehicle to enter the parking area is constructed and then tracked to park the vehicle [3]-[5]. Notice that this approach is strongly dependent on the vehicle odometry availability, essential in the tracking phase. On the contrary, the so called skill-based approaches - mostly based on fuzzy logic controllers [8]-[10] - tries to mimic the manoeuvre of a skilled driver without the need for a prior planning phase. In [8] an odometry based fuzzy logic controller for parallel parking of an AGV vehicle is presented, exploiting distance measures from ultrasonic sensors. In [9], a fuzzy logic based parallel and perpendicular autonomous parking algorithm is derived, that does not need any odometry information. However, it needs a precise estimate of the relative position between the vehicle and parking area, and thus the systems relies on sensor fusion of ultrasonic sensors and a calibrated camera. Finally, in [10], a reverse parallel parking strategy based only on measurements from three sonar sensors placed on the front left corner of the car is presented, with no need for odometry/mapping informations, based on a neuro-fuzzy controller trained with polynomial reference paths. However, possible rear and lateral collisions during the manoeuvre are not considered, making the strategy suitable for the only case of parallel parking beside a single obstacle.

In this work, an odometry free forward perpendicular parking strategy between two obstacles for a light vehicle with an active steer is proposed, based on a frontal low resolution solid state Lidar sensor only. The latter is very similar (for what concerns both performance and cost) to arrays of ultrasonic sensors used for park assist systems on the market. The system is designed for situations where the vehicle has to be parked into a very narrow area between two obstacles - e.g. two vehicles, a vehicle and a wall, etc. - so that it would be 
uncomfortable, even if not impossible, for the driver to get off. An example of the considered light vehicle is reported in Figure 1: the wide (compared to the vehicle dimensions) doors limit the parking of such vehicle between two close obstacles, which in a dense urban scenario is a desirable possibility, especially for this kind of transport.

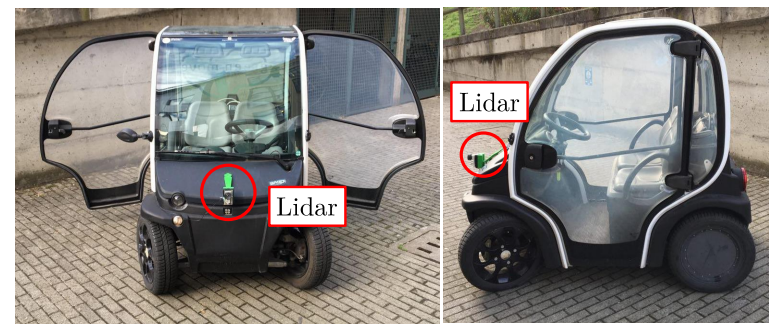

Fig. 1: An example of light electric vehicle, for which the proposed strategy is designed.

The particular use case scenario and hardware limitations make the problem slightly different compared to the literature ones previously described. First of all, due to the non availability of odometry information, an initial scanning/mapping of the parking area cannot be performed and, of course, path planning and tracking techniques must be discarded. Thus, we have not any initial clue on the parking dimensions and feasibility of the manoeuvre. Furthermore, the lack of a priori information, combined with the fact that we can rely only on the front Lidar sensor measurements, turns the vehicle pose estimation during the manoeuvre into a non-trivial problem. Finally, since we can not exploit reverse motion, the final position must be reached by a single forward manoeuvre, without possibility of recover if the vehicle gets stuck against an obstacle.

The remainder of the paper is organized as follows. In Section II, the experimental setup is described, and the objectives and simplifying assumptions are explained. In Section III a modelling for the vehicle low speed motion, as well as the Lidar sensor and the parking environment are derived. In Section IV, the autonomous parking is described. Finally, in Section $\mathrm{V}$ the parking algorithm performances are assessed both in simulation and experimentally.

\section{PRoblem DEFinition}

\section{A. Experimental Setup}

The vehicle considered features the following characteristics:

- Active steering assembly: thanks to a steering DC motor, the steering angle can be autonomously controlled. The control loop is closed thanks to a rotary position encoder and the steering angle is saturated, by mechanical design, to $\pm 40[\mathrm{deg}]$;

- Longitudinal control: the longitudinal motor is driven to run the vehicle only at a nominal speed. Given that no odometry is available an additional wheel encoder has been mounted on the vehicle for validation purposes only;
- Front Lidar: a low resolution solid-state Lidar based on the Time of Flight principle with an horizontal Field Of View (FOV) of $100[\mathrm{deg}]$ - discretized in eight sectors of 12.5 [deg] each - and a vertical FOV of 3 [deg]. Each sector, with a refresh rate of $10 \mathrm{~Hz}$, returns the distance of the closest object that lies in the sector itself, with an accuracy of $5 \mathrm{~cm}$. The sensor is mounted on vehicle front with null pitch angle, pointing perfectly towards, at an height of $60[\mathrm{~cm}]$ (Figure 1) in order to avoid ground detection.

\section{B. Objectives and Problem Description}

The objective of this paper is the development of an automatic perpendicular parking system for light urban vehicles. Due to the previously described limitations, some simplifying assumption on the manoeuvre and environment structure must be done, to guarantee the feasibility of the parking strategy.

We suppose the parking slot to have a pseudo-rectangular shape. The two lateral edges of the parking area are defined by the two obstacles and have similar length. A third obstacle limits the depth of the parking area. The obstacles composing the parking spot are the only ones located in the environment. We assume the vehicle to start the manoeuvre being oriented towards the parking spot, with the latter completely contained into the Lidar FOV. The manoeuvre has to be performed with the vehicle proceeding at a constant (low) longitudinal speed value. Finally, due to the lack of knowledge on the parking spot dimensions, the feasibility of the manoeuvre can not be assessed a priori, but an arrest is commanded if the risk of a collision with obstacles is considered too high.

\section{SYSTEM MODEL}

Based on the assumptions made in Section II-B, the system has been modelled as follows. Three different subsystems are isolated: the vehicle, the parking environment and the Lidar sensor.

Starting from the vehicle, the simple kinematic bicycle model described in [11], [12] is employed: given the low speeds and the kinematics of the steer, the bicycle model still proves to be effective (as the comparison between the simulated and the experimental trajectories will enforce). The following nonlinear equations describe the model dynamics, where the longitudinal speed $v$ is supposed fixed:

$$
\left\{\begin{array}{l}
\dot{x}(t)=v \cdot \cos (\psi(t)+\beta(t)) \\
\dot{y}(t)=v \cdot \sin (\psi(t)+\beta(t)) \\
\dot{v}(t)=0 \\
\dot{\psi}(t)=\left(v / l_{r}\right) \cdot \sin (\beta(t)) \\
\beta(t)=\tan ^{-1}\left(\frac{l_{r}}{l_{r}+l_{f}} \cdot \tan (\delta(t))\right)
\end{array}\right.
$$

In the above equations, $(x, y)$ are the coordinates of the center of mass referred to the inertial frame $(X, Y), \beta$ is the sideslip angle, $\psi$ is the yaw angle between the inertial reference and the vehicle reference frame $\left(x_{v}, y_{v}\right)$, and $l_{f}, l_{r}$ are the distances between the vehicle center of mass and the front and rear axles 

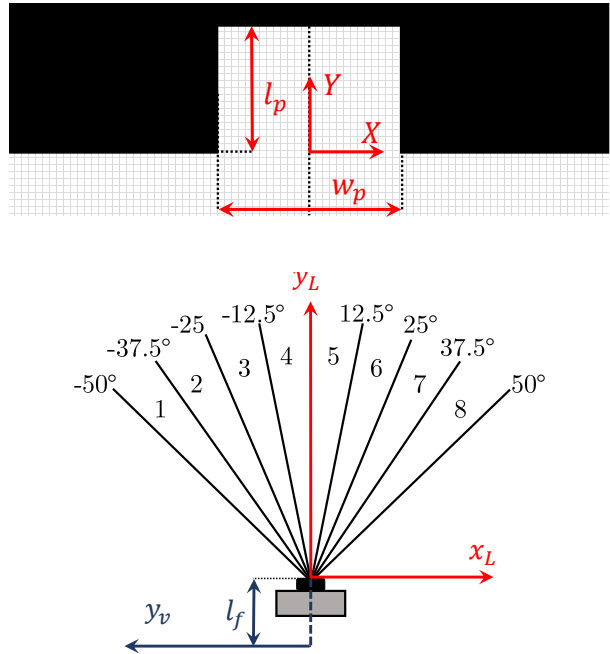

Fig. 2: System modelling schemes: example of binary map parking area (top) and Lidar measurement scheme (bottom).

respectively. The only input to the system is given by the steer angle $\delta$.

Regarding the parking environment, an easy and functional way to represent it is to exploit a $2 \mathrm{D}$ binary occupancy map: an example of is drawn in Figure 2a, where obstacles are represented in black and free space in white. The proposed representation matches the hypothesis previously discussed in Subsection II-B. The available area is characterized by a certain width $w_{p}$ and equal sides length $l_{p}$, while the origin of the $(X, Y)$ frame is conventionally located at the intersection between the longitudinal symmetry axis and the external edge of the parking spot.

Finally, in Figure $2 \mathrm{~b}$ the measurement scheme is represented. The Lidar reference frame $\left(x_{L}, y_{L}\right)$ is located at the front tip of the vehicle, facing the forward longitudinal direction. At each time instant $t$ the device returns eight different measures $d_{i}(t), i=1, \ldots, 8$, one for each available sector, which obviously depend on the position $(x(t), y(t))$ and orientation $\psi(t)$ of the vehicle in the parking environment. Each measure $d_{i}(t)$ reflects the distance of the obstacle that occupies that particular sector: being impossible to determine its exact location within the sector itself, the obstacle is conventionally positioned on the angle bisector, at distance $d_{i}(t)$ from the Lidar frame origin. Thus, eight couples $\left(d_{i}(t), \alpha_{i}\right), i=1, \ldots, 8$, are obtained, each one defining the polar coordinates of the obstacle found in the $i-t h$ sector, where $\alpha_{i}$ takes the following values:

$$
\alpha_{i}=-43.75+12.5 \cdot(i-1)[\mathrm{deg}], \quad i=1, \ldots, 8
$$

The conversion of such measures from polar $\left(d_{i}(t), \alpha_{i}\right)$ to cartesian coordinates $\left(x_{L}^{i}(t), y_{L}^{i}(t)\right)$ in the Lidar reference frame reads:

$$
\left\{\begin{array}{l}
x_{L}^{i}=d_{i}(t) \cdot \sin \alpha_{i} \\
y_{L}^{i}=d_{i}(t) \cdot \cos \alpha_{i} .
\end{array}\right.
$$

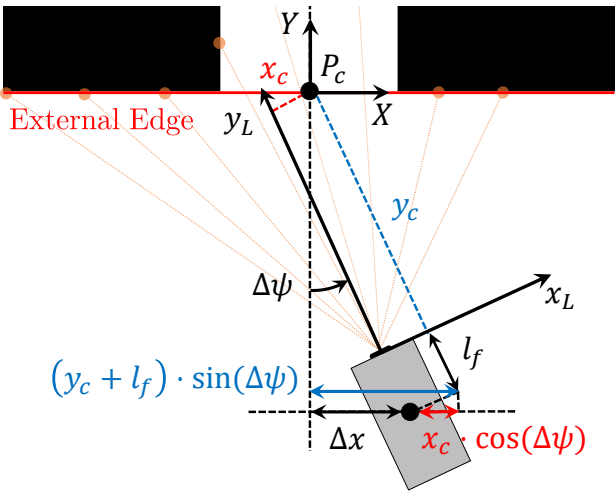

Fig. 3: Definition of the two errors $\Delta \psi$ and $\Delta x$.

\section{Autonomous Parking Strategy}

The autonomous parking strategy computes the steering angle $\delta_{r}(t)$ and the longitudinal speed command for the respective low-level controllers. Such strategy exploits two different algorithms depending on the relative position of the vehicle w.r.t. the parking area. The discriminating factor between the two situations is whether or not the two parking spot external corners are inside the Lidar FOV (i.e. the vehicle is far from or close to the parking spot). In the following, the two different strategies are explained in details.

\section{A. Steer Reference Generation - Far From Parking Area}

When the vehicle is far from the parking area, the goal of the parking strategy is to align it w.r.t. the longitudinal axis of symmetry of the parking spot. Two quantities can thus be defined: the yaw error $\Delta \psi$, as the angular displacement between the vehicle reference axis $y_{v}$ and the parking area symmetry axis (see Figure 3 ), and the lateral error $\Delta x$, as the lateral displacement in the $X$ direction between the parking symmetry axis and the vehicle center of mass. Given these quantities, similarly to the approach in [13], the control law is computed as follows:

$$
\delta_{r}(t)=K_{x} \cdot \Delta x(t)-K_{\psi} \cdot \Delta \psi(t)
$$

where $K_{\psi}$ and $K_{x}$ are positive control gains to be tuned. The main challenge is to retrieve values of $\Delta \psi$ and $\Delta x$ from the sole Lidar measurement. To accomplish this task, the a priori knowledge on the parking spot structure is exploited.

In Figure $4 \mathrm{a}$, an example of measurements in the Lidar frame at a certain time $t$ is depicted. When the vehicle is approaching the parking spot from far distance, its relative orientation $\Delta \psi$ w.r.t. the parking area can be retrieved from the orientation (in the Lidar frame) of the external parking edge defined by the two obstacles. The first step is thus to identify the parking edge from the available Lidar data. To this end, the Convex Hull of the Lidar detections set $\operatorname{Conv}(\mathscr{D})$, where $\mathscr{D}=\left\{P_{L}^{1}, \ldots, P_{L}^{8}\right\}$ is computed (see Figure $4 \mathrm{~b}$ ). Notice that $\operatorname{Conv}(\mathscr{D})$ is a convex polygon with $n_{L} \in[3,8]$ sides, each defined by $S_{L}^{j}: y_{L}=m_{L}^{j} \cdot x_{L}+q_{L}^{j}, j=1, \ldots, n_{L}$ in the Lidar frame, where $m_{L}^{j}$ is the slope and $q_{L}^{j}$ the intercept. As a first 
guess, the external parking edge is identified by one of the $n_{L}$

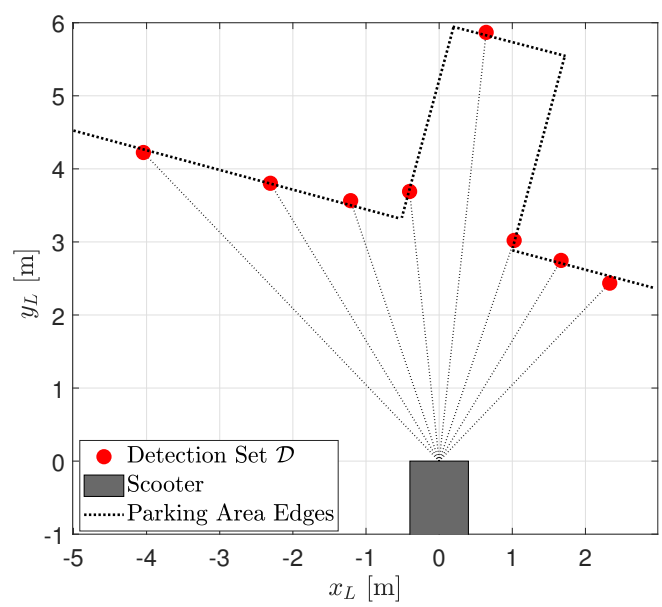

(a) Example of Lidar measurement outside the parking spot.

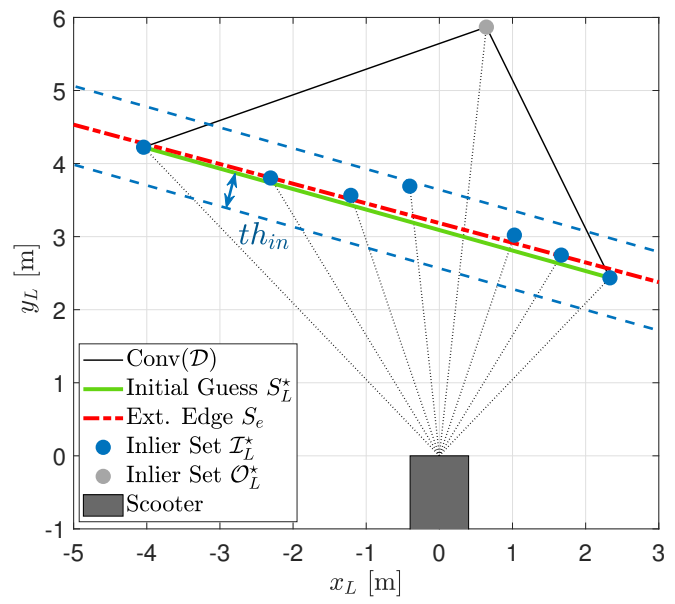

(b) Parking area external edge extraction.

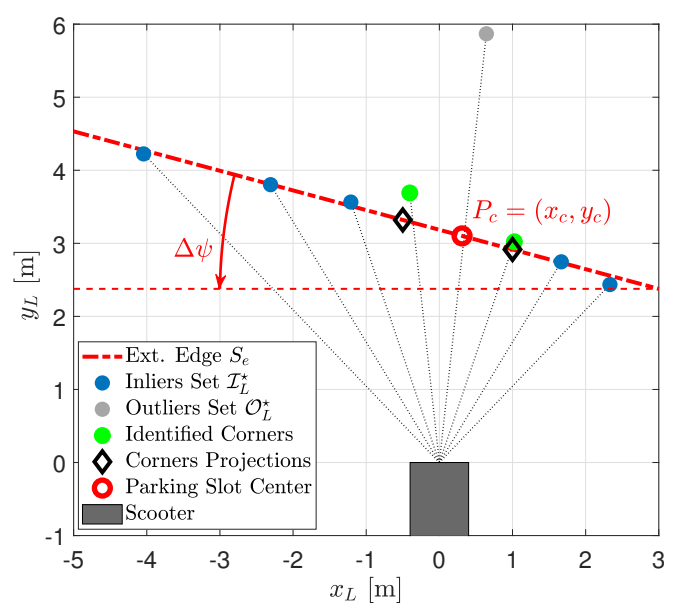

(c) Parking center extraction.

Fig. 4: Steps of the automatic parking strategy to retrieve the yaw error $\Delta \psi$ and the lateral displacement error $\Delta x$ starting from Lidar measurements, when the vehicle is far from the parking area. sides of the convex polygon. The choice is done according to the following steps.

First, given the parking manoeuvre scenario considered, in between the parking edge and the vehicle tip there shouldn't be any other obstacle. Such requirement is mathematically expressed as:

$$
S_{L}^{j} \mid y_{L}^{i} \geq m_{L}^{j} x_{L}^{i}+q_{L}^{j}, P_{L}^{i} \in \mathscr{D}
$$

that enforces the condition that $S_{L}^{j}$ can represent the external edge if and only if there are no detections between it and the vehicle front. $\mathscr{E}$ indicates the set of Convex Hull sides that satisfy such condition.

For each convex hull side $\in \mathscr{E}$, the Lidar detections are divided in two sets, the Inlier $\mathscr{I}_{L}^{j}$ and the Outlier $\mathscr{O}_{L}^{j}$, according to:

$$
\begin{aligned}
\mathscr{I}_{L}^{j} & =\left\{P_{L}^{i} \in \mathscr{D} \mid r_{i j} \leq t h_{i n}, S_{L}^{j} \in \mathscr{E}\right\} \\
r_{i j} & =\frac{\left|m_{L}^{j} x_{L}^{i}-y_{L}^{i}+q_{L}^{j}\right|}{\sqrt{1+\left(m_{L}^{j}\right)^{2}}}
\end{aligned}
$$

In other words, $P_{L}^{i} \in \mathscr{I}_{L}^{j}$ if the Euclidean distance between $P_{L}^{i}$ and $S_{L}^{j}$ is smaller than a given threshold $t h_{i n}$. Otherwise, $P_{L}^{i} \in \mathscr{O}_{L}^{j}$;

The Convex Hull side $S_{L}^{\star} \in \mathscr{E}$ used as external parking edge initial guess is the one that minimizes:

$$
S_{L}^{\star}=\underset{S_{L}^{j} \in \mathscr{E}}{\operatorname{argmin}}\left\{\frac{1}{n_{I}^{2}} \sum_{P_{L}^{i} \in \mathscr{I}_{L}^{j}} r_{i j}\right\}
$$

which is the average euclidean distance value weighted on the number of inliers $n_{I}=\left|\mathscr{I}_{L}^{j}\right|$. In Figure $4 \mathrm{~b}$, the complete procedure is shown in the simplified case of unique convex hull side candidate.

Once $S_{L}^{\star}$ is found, a refinement of its location is performed by a least-square fitting over the inlier set $\mathscr{I}_{L}^{\star}$. The resulting line, having equation $S_{e}: y_{L}=m_{e} \cdot x_{L}+q_{e}$ in the Lidar frame, is the actual identified external parking edge $S_{e}$. The orientation error can now be retrieved (see Figure 4c): $\Delta \psi=-\tan ^{-1}\left(m_{e}\right)$. To compute the displacement error $\Delta x$, the parking spot longitudinal symmetry axis must be found. By definition, this axis is set to be perpendicular to the parking external edge, intersecting the latter at the parking spot centre $P_{c}$ (see Figure 3$)$. Hence, the problem reduces to retrieve the centre coordinates $P_{c}=\left(x_{c}, y_{c}\right)$ in the Lidar frame. These are found starting from the definitions of the inlier and outlier sets $\mathscr{I}_{L}^{\star}$, $\mathscr{O}_{L}^{\star}$. Intuitively, $\mathscr{I}_{L}^{\star}$ represents the set of Lidar detections that belongs to the parking external edge, while $\mathscr{O}_{L}^{\star}$ contains the detections relative to the internal parking area. Thus, park corners are identified as two inliers separated by a group of consecutive outliers (see Figure 4c). The previous intuition can be formalized as follows. A set of candidates external corners couples is defined as:

$$
\mathscr{C}=\left\{\left(P_{L}^{i}, P_{L}^{j}\right) \in \mathscr{I}_{L}^{\star} \mid i<j, P_{L}^{k} \in \mathscr{O}_{L}^{\star} \forall k \in(i, j)\right\}
$$

If more than two candidate couples are found, the couple $\left(P_{L}^{i}, P_{L}^{j}\right)$ which has the minimum sum of the detection distances 


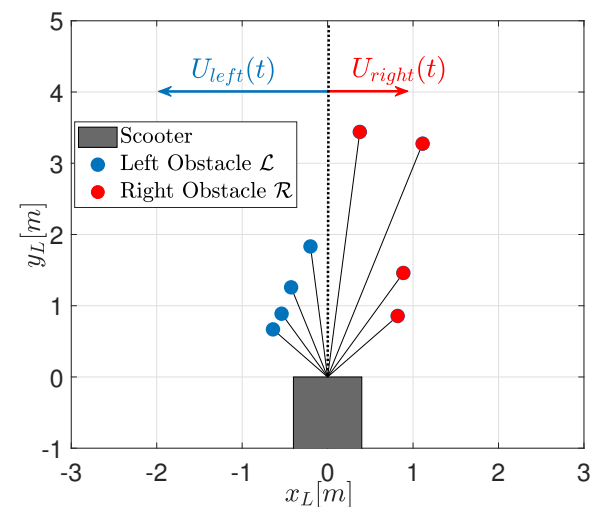

Fig. 5: Scheme of left and right potential fields calculation starting from Lidar measurements.

from the vehicle front is chosen. The center of the parking $P_{c}=\left(x_{c}, y_{c}\right)$ slot is then computed as the midpoint of the projections of the corners on the parking edge $S_{e}$ (see Figure $4 c$ ). Finally, the lateral displacement error $\Delta x$ is obtained by means of trigonometric relations (see the scheme in Figure 3):

$$
\Delta x=\left(y_{c}+l_{f}\right) \cdot \sin \Delta \psi-x_{c} \cdot \cos \Delta \psi
$$

If no center $P_{c}=\left(x_{c}, y_{c}\right)$ is identified instead - e.g. during the manoeuvre one of the parking corners exits the Lidar FOV - $\Delta x$ is set to zero. This way, the vehicle corrects only its orientation, trying to locate again the parking spot at the next iteration.

\section{B. Steer Reference Generation - Close To Parking Area}

While the vehicle approaches the parking spot, at a certain location the external edges of the obstacles exit the Lidar FOV, and thus the $\Delta \psi$ values computed with the previously discussed algorithm become meaningless. Hence, a change of strategy is required.

The first problem to be assessed is how to trigger the strategy switching. The applied rule is to monitor the external sector distance values: in fact, as the vehicle moves forward, the parking edges gradually move towards the outer sector of the Lidar FOV (see Figure 2b) and get closer to the vehicle tip. Defining a threshold $d_{s}$, the switching condition used to identify the entrance in the parking area is thus $d_{i}(t) \leq d_{s}$, where $i \in\{1,2,7,8\}$.

Now, the control goal becomes to reach the bottom end the parking slot, avoiding crashes with the lateral obstacles. The problem is addressed in the obstacle avoidance framework, exploiting Artificial Potential Fields [14], [15]. Firstly, for each Lidar detection $P_{L}^{i}(t)$, a repulsive potential field $U_{i}(t)$ is defined:

$$
U_{i}(t)=\frac{1}{2} K_{u} \cdot\left(\frac{1}{d_{i}(t)}\right)^{2}
$$

where $K_{u}$ is a tuning parameter.

Then, Lidar detections are divided in two subsets $\mathscr{L}$ and $\mathscr{R}$ :

$$
\begin{aligned}
\mathscr{L}(t) & =\left\{P_{L}^{i}(t) \in \mathscr{D}(t) \mid x_{L}^{i}(t) \leq 0\right\} \\
\mathscr{R}(t) & =\left\{P_{L}^{i}(t) \in \mathscr{D}(t) \mid x_{L}^{i}(t)>0\right\}
\end{aligned}
$$

associated respectively to the left and right obstacles surrounding the parking area (see Figure 5). The total repulsive fields of left and right obstacles are obtained as:

$$
U_{l}(t)=\frac{1}{|\mathscr{L}(t)|} \sum_{P_{L}^{i} \in \mathscr{L}} U_{i}(t), \quad U_{r}(t)=\frac{1}{|\mathscr{R}(t)|} \sum_{P_{L}^{i} \in \mathscr{R}} U_{i}(t)
$$

Finally, the control action is defined as the difference between the two repulsive fields relative to the left and right obstacles:

$$
\delta(t)=U_{l}(t)-U_{r}(t)
$$

This way, the vehicle moves away from the nearest lateral obstacle, avoiding lateral collisions. Notice that trajectory convergence towards the end of the parking area is ensured by convexity its shape.

\section{Speed Reference Generation}

The parking manoeuvre is performed at low and constant speed. There are two situations where a stop is requested, and in both cases the parking manoeuvre ends: when the vehicle reaches the bottom of the parking spot or if it gets too close to an obstacle.

The first event is triggered when the average distance measured by the four central Lidar sectors is lower than a threshold $\bar{d}_{\text {end }}$ :

$$
\frac{1}{4} \sum_{i=3}^{6} d_{i}(t) \leq \bar{d}_{\text {end }}
$$

The second event instead is detected if one of the Lidar measures is below the minimum safe distance: $\exists d_{i}(t) \in$ $\mathscr{D}(t) \mid d_{i}(t) \leq d_{\text {min }}$.

\section{Simulation AND EXPERIMENTAL RESUlts}

The system model, as well as the proposed control strategy, has been implemented in Matlab/Simulink software environment, to verify its correct behaviour and tune the parameters. In such setup, the simplified binary parking environment shown in Section III has been reproduced as discretized in cells of fixed dimensions $(e . g .5 \mathrm{~cm} \times 5 \mathrm{~cm}$, to mimic the sensor resolution). Furthermore, to model possible crashes with the obstacles, the vehicle itself is represented as a moving rectangle whose length and width are defined as the maximum dimensions of the vehicle, $l_{\max }$ and $w_{\max }$ respectively.

We first focus on the control parameters tuning. Starting from the two gains $K_{\psi}$ and $K_{x}$, as discussed in [13], a tradeoff arises in their choice. Considering the ratio $K_{\psi} / K_{x}$, a too high value leads to an early reduction of the orientation error, thus heading the vehicle against one of the lateral obstacles. On the contrary, too small values of $K_{\psi} / K_{x}$ yields too high orientation errors causing the parking slot to exit the Lidar FOV, which is in fact an undesired situation.

Similarly, a trade-off exists on the choice of $K_{u}$. Too high values of such parameter lead to oscillatory behaviour of the controlled system, while too low values cause the control action to be insufficient to avoid lateral crashes. Finally, regarding the strategy switching parameter $d_{s}$, a conservative tuning so as to obtain an early switch has to be preferred w.r.t 


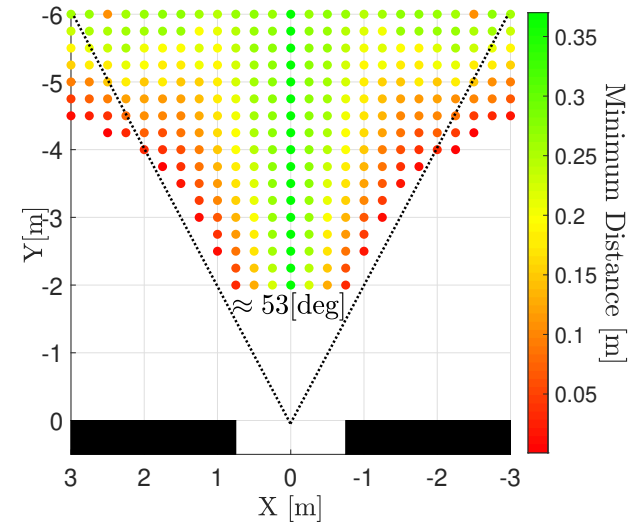

Fig. 6: Simulation assessment of the algorithm performance, the vehicle starts the manoeuvre oriented towards the parking spot centre.

a late switch between the far and near parking strategies. In the latter case, the wrong detection of the parking edge will result in orientation reference errors which can lead the vehicle towards the obstacles.

The parameter tuning has been performed in the simulation environment: for a given set of parameters $\left(K_{\psi}, K_{x}, K_{u}, d_{s}\right)$ several (namely 425) parking simulations were performed, from from different positions. Considering the length of the vehicle and the 2 meters maximum curvature trajectory (due to the steer angle limitations), the initial condition range of interest has been considered to be $x \in[-3,3]$ and $y \in[-2,-6]$. Moreover, an initial vehicle orientation pointing towards the parking area centre has been set. Then, the following cost function has been computed:

$$
J=\sum_{j=1}^{425} \bar{d}_{j} \quad \text { where } \quad \bar{d}_{j}=\min d_{i}(t), \quad i \in 1, \cdots, 8
$$

which represents the sum of the minimum distances from the obstacles for each simulated parking manoeuvre. If, during a simulation, the vehicle was not able to successfully complete the manoeuvre, the minimum distance has been set equal to zero. The best control parameters set $\left(K_{\psi}, K_{x}, K_{u}, d_{s}\right)$ is found maximizing the proposed cost function. The overall assessment of the simulated performances, with the obtained tuning, is shown in Figure 6. Each dot represents a vehicle initial position and its colour is related to the value of $\bar{d}_{j}$ accomplished during the simulation; missing dots represent initial conditions of unsuccessful parking manoeuvres. The proposed tuning is capable of producing a feasible and safe parking manoeuvre when the vehicle initial yaw angle is in the interval $\psi \in[-25,25] \mathrm{deg}$. As expected, performances improve for higher initial distances from the parking spot, due to the greater room available for the manoeuvre. The main limitation to the parking feasibility is due to the fact that the vehicle, to recover its orientation and lateral error, should follow a trajectory that causes the parking slot to exit the FOV of the Lidar.

\begin{tabular}{cccc} 
Parameter & Symbol & Value & UM \\
\hline \multicolumn{4}{c}{ System Model } \\
\hline$l_{f}$ & Front Axle Length & 0.75 & {$[\mathrm{~m}]$} \\
$l_{r}$ & Rear Axle Length & 0.75 & {$[\mathrm{~m}]$} \\
$l_{\max }$ & Total Lenght & 2.15 & {$[\mathrm{~m}]$} \\
$w_{\max }$ & Total Width & 0.8 & {$[\mathrm{~m}]$} \\
\hline \multicolumn{4}{c}{ Parking Area } \\
\hline$l_{p}$ & Park Area Length & 2.5 & {$[\mathrm{~m}]$} \\
$w_{p}$ & Park Area Width & 1.5 & {$[\mathrm{~m}]$} \\
\hline \multicolumn{4}{c}{ Control Strategy } \\
\hline $\bar{v}$ & Forward Speed & 1 & {$[\mathrm{Km} / \mathrm{h}]$} \\
$t_{\text {in }}$ & Inlier Threshold & 0.4 & {$[\mathrm{~m}]$} \\
$K_{\psi}$ & $\psi$ Error Gain & 45 & {$[-]$} \\
$K_{x}$ & $x$ Error Gain & 20 & {$[\mathrm{deg} / \mathrm{m}]$} \\
$K_{u}$ & Pot. Field Gain & 30 & {$\left[\mathrm{deg} / \mathrm{m}^{2}\right]$} \\
$d_{s}$ & Switch Distance & 1.5 & {$[\mathrm{~m}]$} \\
$\bar{d}_{\text {end }}$ & End Park Distance & 0.3 & {$[\mathrm{~m}]$} \\
$d_{\min }$ & Minimum Distance & 0.2 & {$[\mathrm{~m}]$}
\end{tabular}

TABLE I: Overall model and control parameters.

The values for all the simulation parameters are summarized in Table I, where a standard light vehicle parking spot dimensions (regarding Italian legislation [16]) have been considered.

The proposed control strategy has been implemented on the real system and validation experiments have been conducted reproducing the simplified parking scenario using cardboard boxes. Two examples of simulated and real trajectories (reconstructed thanks to the accessorial odometry) starting from two different initial poses are depicted in Figure 7. It is worth to notice the good matching between the simulated and real paths followed by the vehicle in both cases. This result confirms the validity of the proposed modelling strategy in reproducing the behaviour of the real system and the effectiveness of the simulation based parameter tuning. Furthermore, it can be seen how the trajectory produced by the algorithm are smooth and not oscillating (thus not stressing the steering mechanism), thanks to a correct choice of the control gains. Looking at the two tests individually, in Figure 7 a the vehicle starts the manoeuvre pointing the center of the parking spot with an initial position at the edge of the feasibility area $\left(x_{i}=2[\mathrm{~m}]\right.$, $\left.y_{i}=-4[\mathrm{~m}]\right)$, and initial inclination of 26 [deg]. During the first phase of the strategy (Section IV-A) the algorithm reduces the lateral displacement $\Delta x$ w.r.t. the parking spot axis, keeping bounded the value for $\Delta \psi$. As the strategy switch occurs (i.e. when the vehicle front reaches the blue dot), the behaviour slightly changes and the algorithm reduces also the vehicle angular error in order to avoid collisions with the obstacles. Finally, the vehicle enters the parking area and arrests its motion when the parking spot end is detected. During the manoeuvre, the minimum distance from the obstacles is $4[\mathrm{~cm}]$, which is small but yet not critical, especially when compared the the overall available parking space.

Figure $7 \mathrm{~b}$, shows the parking manoeuvre in a different scenario, where the vehicle in the initial configuration does not point towards the center of the parking slot (which is still inside the Lidar FOV). The minimum distance from obstacles in this case is $12[\mathrm{~cm}]$. 


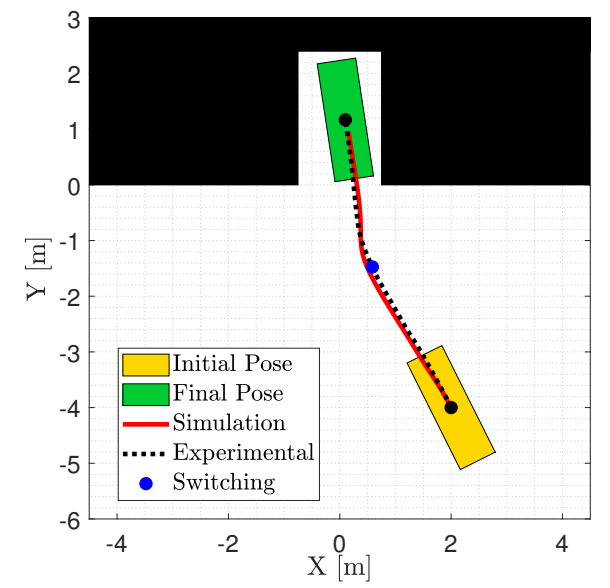

(a) Simulated and experimental parking manoeuvre starting from the initial pose: $x_{i}=2[\mathrm{~m}], y_{i}=-4[\mathrm{~m}], \psi_{i}=26[\mathrm{deg}]$.

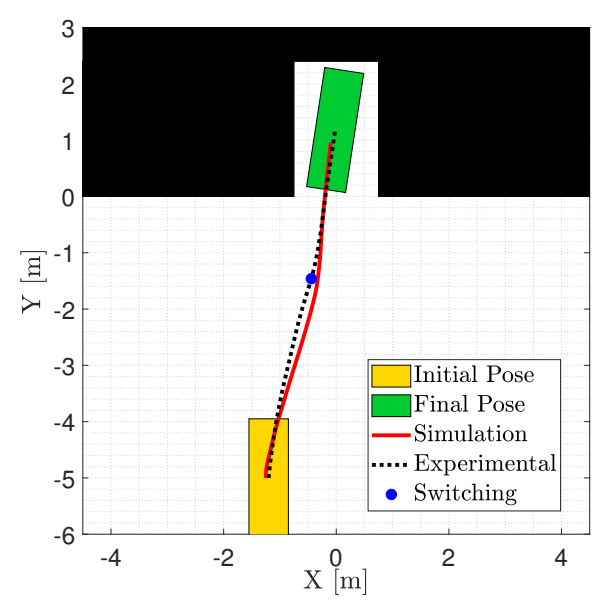

(b) Simulated and experimental parking manoeuvre starting from the initial pose: $x_{i}=-1.2[\mathrm{~m}], y_{i}=-5[\mathrm{~m}], \psi_{i}=0[\mathrm{deg}]$.

Fig. 7: Comparison between simulated and real parking trajectories considering two different vehicle initial poses.

\section{CONCLUSIONS}

In this paper an odometry-free parking strategy meant for light urban vehicles, based on a low resolution Lidar, has been presented. The core of the algorithm is the inclusion of a priori information about the parking area which is structured in a specific - though realistic - way, allowing to retrieve the vehicle orientation and position w.r.t. the parking area. Experimental results show the effectiveness of such strategy, allowing for a wide area of initial vehicle positions that result in a collisionfree parking.

\section{ACKNOWLEDGEMENTS}

The authors acknowledge Mario Santucci and Onorino Di Tanna, from Piaggio Group, for their support to the work.

\section{REFERENCES}

[1] W. Wang, Y. Song, J. Zhang, and H. Deng, "Automatic parking of vehicles: A review of literatures," International Journal of Automotive Technology, vol. 15, no. 6, pp. 967-978, oct 2014.
[2] Y. Song and C. Liao, "Analysis and review of state-of-the-art automatic parking assist system," Proceedings - IEEE International Conference on Vehicular Electronics and Safety, ICVES 2016, pp. 61-66, 2016.

[3] S. H. Jeong, C. G. Choi, J. N. Oh, P. J. Yoon, B. S. Kim, M. Kim, and K. H. Lee, "Low cost design of parallel parking assist system based on an ultrasonic sensor," International Journal of Automotive Technology, vol. 11, no. 3, pp. 409-416, jun 2010.

[4] T. H. Hsu, J. F. Liu, P. N. Yu, W. S. Lee, and J. S. Hsu, "Development of an automatic parking system for vehicle," 2008 IEEE Vehicle Power and Propulsion Conference, VPPC 2008, pp. 1-6, 2008.

[5] K. Jiang and L. Seneviratne, "A sensor guided autonomous parking system for nonholonomic mobile robots," in Proceedings IEEE International Conference on Robotics and Automation, vol. 1. IEEE, 1999, pp. 311-316.

[6] W. J. Park, B. S. Kim, D. E. Seo, D. S. Kim, and K. H. Lee, "Parking space detection using ultrasonic sensor in parking assistance system," IEEE Intelligent Vehicles Symposium, pp. 1039-1044, 2008.

[7] J. K. Suhr and H. G. Jung, "Sensor fusion-based vacant parking slot detection and tracking," IEEE Transactions on Intelligent Transportation Systems, vol. 15, no. 1, pp. 21-36, 2014.

[8] Y. Zhao and E. G. Collins, "Robust automatic parallel parking in tight spaces via fuzzy logic," Robotics and Autonomous Systems, vol. 51, no. 2-3, pp. 111-127, 2005.

[9] Y. Ryu, S. Oh, and S. Kim, "Robust Automatic Parking without Odometry using an Evolutionary Fuzzy Logic Controller," I. J. of Control, Automation and Systems, vol. 6, no. 3, pp. 434-443, 2008.

[10] K. Demirli and M. Khoshnejad, "Autonomous parallel parking of a carlike mobile robot by a neuro-fuzzy sensor-based controller," Fuzzy Sets and Systems, vol. 160, no. 19, pp. 2876-2891, 2009.

[11] J. Kong, M. Pfeiffer, G. Schildbach, and F. Borrelli, "Kinematic and dynamic vehicle models for autonomous driving control design," in IEEE Intelligent Vehicles Symposium (IV), 2015, pp. 1094-1099.

[12] P. Polack, F. Altché, A. De La, A. De La Fortelle, and A. de La Fortelle, "The Kinematic Bicycle Model: a Consistent Model for Planning Feasible Trajectories for Autonomous Vehicles?" in IEEE Intelligent Vehicles Symposium (IV), 2017.

[13] O. Amidi and C. Thorpe, "Integrated mobile robot control," in Proc. SPIE 1388, Mobile Robots V, 1991.

[14] O. Khatib, "Real-time obstacle avoidance for manipulators and mobile robots," in Proceedings. 1985 IEEE International Conference on Robotics and Automation, vol. 2. Institute of Electrical and Electronics Engineers, 1985, pp. 500-505.

[15] Min Cheol Lee and Min Gyu Park, "Artificial potential field based path planning for mobile robots using a virtual obstacle concept," in Proceedings 2003 IEEE/ASME International Conference on Advanced Intelligent Mechatronics (AIM 2003), vol. 2, 2003, pp. 735-740.

[16] M. delle Infrastrutture e dei Trasporti, Italian Traffic Laws, Decreto Legislativo 30 Aprile 1992 n.285, 1992. 